\title{
Effect of Long-Term Formalin Preservation on Bending Properties and Fracture Toughness of Bovine Compact Bone
}

\author{
Hisao Kikugawa ${ }^{1}$ and Takashi Asaka ${ }^{2}$ \\ ${ }^{1}$ Department of Mechanical Engineering, School of Engineering II, Tokai University, Tokyo 151-0063, Japan \\ ${ }^{2}$ Department of Applied Chemistry, School of Engineering, Tokai University, Kanagawa 259-1292, Japan
}

\begin{abstract}
To precisely determine the mechanical properties of a bone, the effects of the preservation method on its mechanical properties need to be minimized. It seems likely that prolonged exposure to formalin (formaldehyde solution) will have some affect the mechanical properties of bone. This study investigated the effect of the formalin fixation method on the bending properties and fracture toughness of bovine cortical bone after short-term and relatively long-term preservation. To determine the elements and the quantities eluted from bone into formalin solution (the preservation medium), qualitative and quantitative analyses were performed by inductively coupled plasma atomic emission spectroscopy (ICPAES). Formalin fixation augments the bending stiffness of the cortical bone while diminishing its fracture toughness. As the bending strength diminishes concurrently with removal of $\mathrm{Ca}^{2+}$, this implies that inorganic components of bone contribute to its bending strength.
\end{abstract}

(Received June 8, 2004; Accepted August 17, 2004)

Keywords: bone, mechanical properties, fracture toughness, mechanical test, formalin preservation, eluted element analysis

\section{Introduction}

To precisely determine the mechanical properties of a bone, the effects of the preservation method on its mechanical properties need to be minimized. However, bone specimens have not necessarily been preserved by a standardized method. In studies evaluating the material properties of various bones, deep freeze preservation or chemical fixation using reagents such as formalin (formaldehyde aqueous solution) or ethanol is often carried out on sample pieces of bone for the purpose of antisepsis and sterilization.

Studying the effect of antisepsis on cortical bone, McElhaney et al. ${ }^{1)}$ examined an antiseptic solution containing alcohol and glycerin and reported that the ultimate compressive strength of the cortical bone decreased by $12 \%$, whereas the values for tensile properties decreased only slightly. By contract, Evans ${ }^{2)}$ reported a significant augmentation in the tensile elastic modulus and the strength of the cortical bone. As a result, the stiffness increased by approximately $16 \%$, while the strength increased by approximately $10 \%$. The effect of ethanol preservation on cancellous bone for a relatively long term (100 days) was examined by Linde et $a l .{ }^{3)}$ and they concluded that compressive stiffness did not change significantly.

Tanaka et ll $^{4)}$ studied changes in the bending property of gallus tibia preserved in $10 \%$ formalin for a certain period of time. They concluded that there was no significant change in stiffness, ultimate load, or absorbed energy after a relatively long preservation period of 60 days.

Currey et $a l .{ }^{5)}$ have reported that formalin fixation of bovine cortical bone for a short term (three hours) does not effect affect its bending or tensile properties, and that chemical fixation of bone using formalin augments the number of inter- and intra-collagenic cross-linkages, since it acts directly on the primary amine of a collagen chain; as a result, at least one of the two essential elements of bone may alter its mechanical properties.

Unlike the freezing method, ${ }^{3,6-10)}$ studies of chemical bone preservation methods provide inconsistent results. There are a few reports which suggest that chemical antisepsis and preservation methods affect the mechanical properties of bone; however, the tissue fixation method used was not specified in these studies. It is therefore uncertain whether the mechanical properties of bone are affected when exposed to formalin for a long period of time.

In this study, the effect of formalin on the bending property and fracture toughness of bovine cortical bone was investigated in both short-term and relatively long-term preservation.

\section{Experimental Methods}

\subsection{Test specimen preparation}

Cortical bone from the shaft of fresh bovine femur was used for the experiment because it has static tension and compression properties similar to those observed in humans. ${ }^{11)}$ In order to ascertain regional differences in the effect of preservation, the cross section of the shaft was quadrisected - anterior, posterior, lateral, and medial - to construct test specimens.

The microscopic structure of bovine femur ${ }^{12)}$ consists of plexiform bone that is dominated on the anterior and lateral sides by nuclei of nonlamellar bone interspersed with lamellar bone. The posterior side is composed of Haversian bone that is mainly dominated by osteon. The medial side consists of a mixture of plexiform bone and Haversian bone. In this study, a scanning electron microscope (SEM) was used to examine the fracture surface perpendicular to the bone axis of test specimens; this revealed that plexiform bone was mostly distributed on the anterior, lateral, and medial sides, whereas Haversian bone was widespread on the posterior side.

Commercially available formaldehyde aqueous solution was diluted 10-fold, and was used as a preservation solution for the test specimens; this solution is commonly known as $10 \%$ formalin. To precisely determine the concentration of formaldehyde, quantitative analysis was performed using iodimetry, which determined the formaldehyde concentration 
to be 3.7 mass\% (hereinafter referred to as formalin preservative). The concentration of formalin used in this study was the same as that used by McElhaney et al. ${ }^{1)}$ (who did not use antisepsis or glycerin) and was approximately the same as that used by Tanaka ${ }^{4)}$ and Currey. ${ }^{5}$ )

The test specimen was maintained in physiological saline for approximately one hour before the mechanical test.

\subsection{The three-point bending test}

Fifteen femurs were used for the three-point bending test, and 6-8 pieces of rectangular-shaped test specimens with dimensions of $3 \mathrm{~mm} \times 3 \mathrm{~mm} \times 20 \mathrm{~mm}(b \times h \times l)$ were prepared by machining the areas as close as possible to each other within each quadrisected region. From each extraction region, 12 to 15 test specimens were randomly prepared, and these were separated into three groups to study the effect of load speed, while another three groups were formed for the study of the effect of formalin preservation.

In order to examine the effect of formalin preservation, three test specimens from each extraction region (anterior, posterior, lateral and medial), and for each preservation group (7 days, 30 days and 140 days), were soaked in $25 \mathrm{~mL}$ formalin preservative and tight sealed, in preparation for the three-point bending test. Test specimens were initially cleansed in an ultrasonic bath with formalin preservative for a minute before they were tight sealed. These preserved specimens were stored in a dark place at room temperature and were used for subsequent tests and analysis.

The three-point bending test of the bone specimens were performed by an Instron-type testing machine with a capacity of $1000 \mathrm{~N}$. The distance between the supporting points (the span length) was $10 \mathrm{~mm}$ and the load was exerted from the outer circumference of the femur towards the interior portion and maintained until fracture of the test specimen occurred. Bending stiffness and bending strength were calculated from the load-displacement curve that was obtained.

The load-displacement relationship indicated that: the toe region pointed in a slightly downward direction in the initial low-load region; the medium-load region exhibited a linear trend; and the toe region pointed in a slightly upward direction in the high-load region. The samples fractured instantaneously at the maximum value. The linear part of the data in the medium-load region was linearly approximated by the least square method and the slope of the curve, $P / \delta$ (load/ displacement), was determined, which was then employed to calculate bending stiffness, $E$, using the following equation: ${ }^{13)}$

$$
E=\frac{(P / \delta) l^{3}}{48 I}\left\{1+2.85(h / l)^{2}-0.84(h / l)^{3}\right\}
$$

where $I$ is the geometric moment of inertia of a beam, $l$ is the span length, and $h$ is the cross-section height of the test specimen.

Bending strength, $\sigma$, was calculated using the load at the fracture, i.e., the ultimate load, $P$, using the following equation:

$$
\sigma=\frac{M}{Z}=\frac{3 P l}{2 b h^{2}}=2 \frac{l}{h} \tau
$$

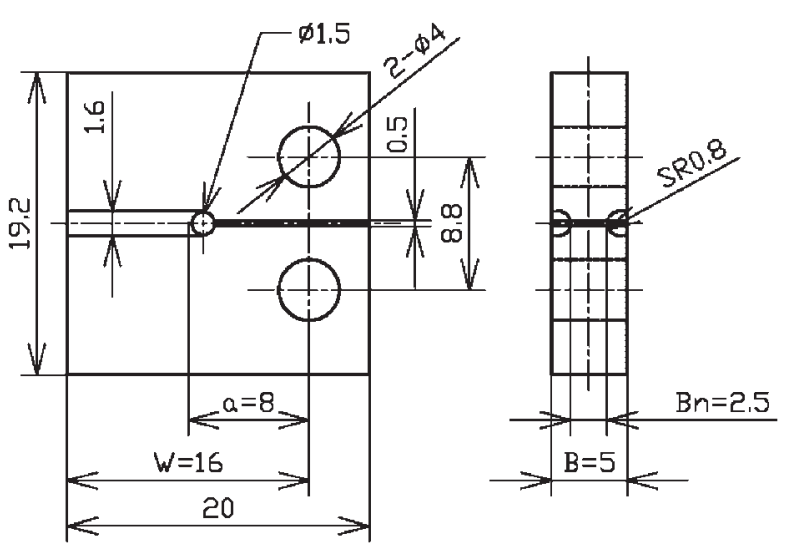

Fig. 1 Compact tension specimen and dimension.

where $Z$ is the section modulus of a beam, $b$ is the crosssection width of the test specimen, and $\tau$ is the shear stress. In this experiment, $\tau$ was set at $\tau=0.15 \sigma$.

\subsection{The fracture toughness test}

A compact tension test specimen as shown in Fig. 1 was prepared according to ASTM E399-9014) and used for the fracture toughness test. A slit was introduced in a direction perpendicular to the bone axis, and its end was shaped as a keyhole. To prevent the development of a crack parallel to the direction of a slit insertion, a U-shaped side groove ${ }^{15)}$ was created. In addition, a $0.2 \mathrm{~mm}$ slit was created from the end of the keyhole slit by a razor.

The transverse section of the femur was quadrisected as in the bending test, and one or two test specimens per region and 12 specimens per group were prepared. The effect of formalin preservation was investigated in 5 groups after 2, 7, 30, 60, and 90 days of preservation.

Fracture toughness, $K_{Q}$, was evaluated using the following equation derived by Norman et al.: $\left.{ }^{15}\right)$

$$
K_{Q}=\frac{P f(a / W)}{\left(B B_{n}\right)^{1 / 2} W^{1 / 2}}
$$

where $P$ is the load, $B$ is the thickness of the test specimen, $B_{n}$ is the thickness of the groove, $W$ is the width of the test specimen, $a$ is the length of the slit, and $f(a / W)$ is the form factor. ${ }^{14)}$ Equipment similar to that used for the three-point bending test was employed for the mechanical test, and the load speed was set at $20 \mathrm{~mm} / \mathrm{min}$. The load-displacement relationship was ASTM Type III, ${ }^{14)}$ and the fracture developed unsteadily with the simultaneous generation of the ultimate load; fracture toughness was evaluated from this ultimate load.

\subsection{Chemical analysis: elements eluted into the preser- vation solution}

In formalin preservation of bone, constituent elemental components such as hydroxyapatite (calcium phosphate salt) - a main constituent of bone — are thought to elute into formalin. By contrast, collagen exists in bone as a polypeptide with a triple-helix structure, and is insoluble in water. The inorganic elements eluted into formalin preservative were analyzed by an inductively coupled plasma atomic emission spectrometer (ICP), with the purpose of examining 
the relationship between the eluted quantities of inorganic components of bone, and the mechanical properties of bone.

The primary elements of bone are $\mathrm{Ca}$ and $\mathrm{P}$ that constitute hydroxyapatite. To analyze which elements elute into formalin preservative, $1 \mathrm{~mL}$ of formalin preservative from the 140 day preservation group (which was expected to have the highest elution concentration) was extracted (region: anterior), and then diluted 50-fold in deionized water; qualitative analysis was then performed by ICP.

Qualitative analysis revealed the existence of $\mathrm{K}$ (Potassium), $\mathrm{Na}$ (Sodium), and $\mathrm{Mg}$ (Magnesium) in addition to $\mathrm{Ca}$ and $\mathrm{P}$, and it was confirmed by the same method that these elements were not present in deionized water, or in the formalin preservative solution.

$\mathrm{Na}$ and $\mathrm{K}$ eluted into formalin preservative were excluded from the analysis, as they are electrolytes in the living body. However, precise quantitative analysis was performed on three elements, $\mathrm{Ca}, \mathrm{P}$ and $\mathrm{Mg}$, since $\mathrm{Mg}$ is an essential element for the metabolism of Ca. Quantitative analysis was performed by ICP, using an appropriate concentration of standard solution for each of $\mathrm{Ca}, \mathrm{P}$, and $\mathrm{Mg}$, and using formalin preservative as a blank sample.

When inorganic elements of bone elute into formalin preservative, the $\mathrm{pH}$ of the preservative will change. Therefore, $\mathrm{pH}$ measurement of formalin preservative was performed using a $\mathrm{pH}$ meter.

\subsection{Statistical analysis}

A statistical procedure ${ }^{16)}$ was used to examine the difference between the averages for each group. One-way analysis of variance was employed to verify the difference of averages for the bending properties with respect to load speed. The significance for the bending property of the control and preserved groups was verified by the $\mathrm{F}$ test to examine homoscedasticity. This was followed by Student's t-test when the distribution was found to be equal; otherwise, the results of Welch's t-test were accepted. A p-value of $<0.05$ was considered to be statistically significant.

\section{Results and Discussion}

In order to examine the effect of load speed, the cross head speed of the material testing machine was altered to 1,20 , and $30 \mathrm{~mm} / \mathrm{min}$ and the bending property was evaluated at each of these speeds. The results are shown in Table 1.

No significant change was observed in bending stiffness for either plexiform bone or Haversian bone. By contrast, the average bending strength at each load speed differed significantly $(\mathrm{p}<0.001)$, and tended to increase as load speed increased. The aim of this study was to compare the extent of the difference in bending property with or without formalin treatment. Therefore, it was desirable to use the same specimens under the same test conditions. Thus, the augmented strength with increased load speed was not an essential problem to consider. The following experiments were performed at a load speed of $20 \mathrm{~mm} / \mathrm{min}$, which enabled completion of each test within three seconds so that the wet condition could be maintained.

The bending stiffness of cortical bone is approximately 5-16 $\mathrm{GPa}^{17,18)}$ while the values obtained in this experiment were lower. This is due to the aspect ratio of 3.3 (span length/ cross sectional height) of the beam used in this experiment, which is lower than that of a standard beam. We conducted another experiment using a test specimen with an aspect ratio of 6.7 , and the bending stiffness was found to be $6-8 \mathrm{GPa}$. Thus, the result of this study is judged to be reasonable. These values of bending strength also correspond well with those reported by Currey ${ }^{5)}$ and Reilly. ${ }^{19)}$

The time-change of bending stiffness is shown in Fig. 2. The ordinate indicates the rate of change, which is obtained by dividing the difference between the averages of the control group and formalin preservation group by the average of control group. Figure 2 indicates that the increase in the bending stiffness in each group is independent of the bone structure and the preservation period, and that the values are higher in plexiform bone than in Haversian bone for each preservation time period. The increase in bending stiffness from the initial stage of preservation was significant; by the 140th day, the increase in the constants for both the plexiform bone and Haversian bone reached $30 \%$.

As described above, blood vessels and collagen exist in bone in addition to the inorganic components. Boskey et al. ${ }^{20)}$ reported that due to formalin fixation, the density of collagen

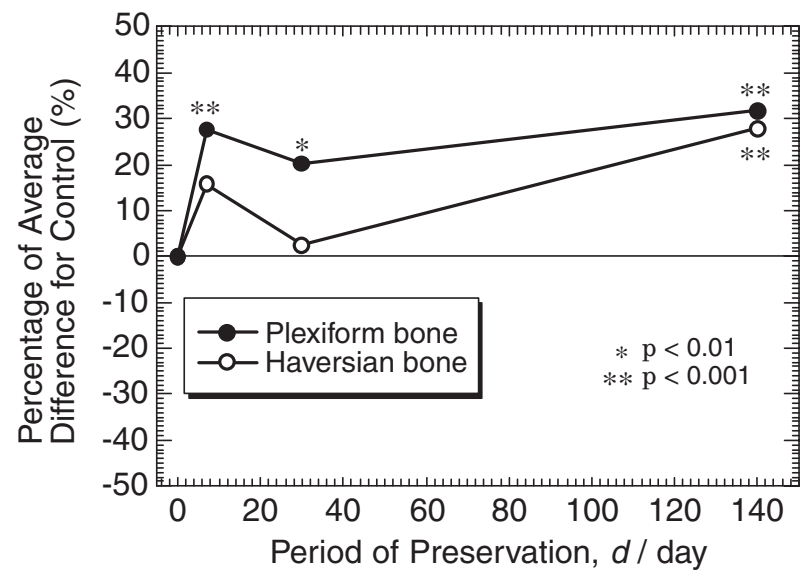

Fig. 2 Bending Stiffness.

Table 1 Effect of cross head speed on the bending test.

\begin{tabular}{|c|c|c|c|c|c|c|}
\hline \multirow[b]{2}{*}{$\begin{array}{c}\text { Cross head speed, } \\
\quad v / \mathrm{mm} \cdot \mathrm{min}^{-1}\end{array}$} & \multicolumn{3}{|c|}{ Haversian bone } & \multicolumn{3}{|c|}{ Plexiform bone } \\
\hline & $n$ & $\begin{array}{l}\text { Stiffness, } \\
E / \mathrm{GPa}\end{array}$ & $\begin{array}{l}\text { Strength, } \\
\sigma / \mathrm{MPa}\end{array}$ & $n$ & $\begin{array}{l}\text { Stiffness, } \\
E / \mathrm{GPa}\end{array}$ & $\begin{array}{l}\text { Strength, } \\
\sigma / \mathrm{MPa}\end{array}$ \\
\hline 1 & 15 & $3.47(0.51)$ & $200.5(24.2)$ & 38 & $3.75(0.54)$ & $222.0(28.5)$ \\
\hline 20 & 12 & $3.51(0.48)$ & $227.2(30.7)$ & 37 & $4.06(0.51)$ & $261.3(16.4)$ \\
\hline 30 & 15 & $3.58(0.37)$ & 240.7 (24.7) & 40 & $4.08(0.45)$ & 263.5 (27.9) \\
\hline
\end{tabular}




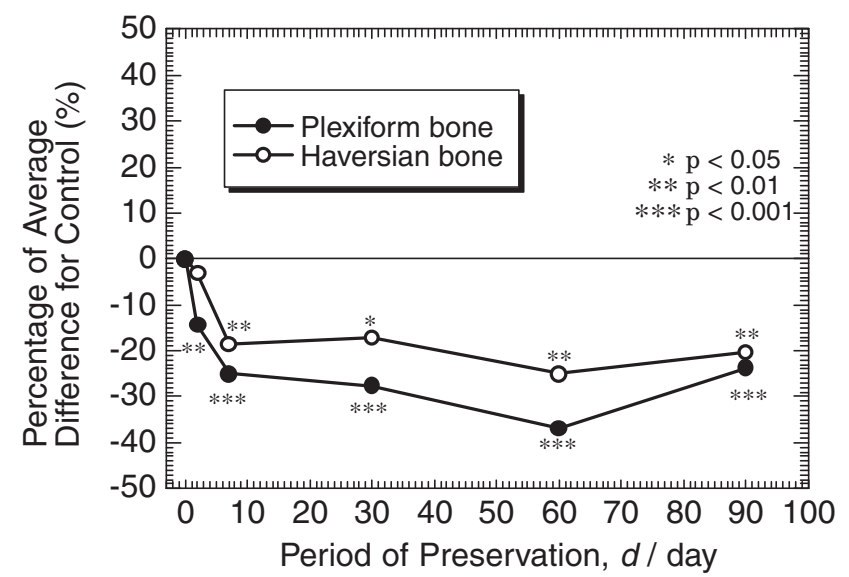

Fig. 3 Fracture toughness.

fibers increased due to increasing cross-linking.

Collagen fibers are dehydrated by formalin. Each polypeptide chain of collagen consisting of a triple-helix structure incorporating amino acids such as glycine, proline, and hydroxyproline, and it can be inferred that the - $\mathrm{OH}$ groups of hydroxyproline and formaldehyde form a cross-link by formalization. The increase in the bending stiffness of bone preserved in formalin could be explained by collagen hardening as described above. The time-change of fracture toughness is shown in Fig. 3, similar to that in Fig. 2. The values for the control group (average \pm S.D.) in this case are $5.32 \pm 0.42 \mathrm{MPa} \cdot \mathrm{m}^{1 / 2}$ for plexiform bone and $4.61 \pm 0.84$ $\mathrm{MPa} \cdot \mathrm{m}^{1 / 2}$ for Haversian bone, which are consistent with previously reported values. ${ }^{21-24)}$

Figure 3 indicates that fracture toughness diminishes more than $20 \%$, immediately after preservation, which is statistically significant, and the values of plexiform bone are higher than those of Haversian bone. The result of ICP analysis implies that with formalin preservation, the elution of elements such as $\mathrm{Ca}, \mathrm{P}$, and $\mathrm{Mg}$ from bone into formalin preservative facilitated the inducement of micro-cracks in the bone, which resulted in the decrease in fracture toughness. In any case, the decrease in fracture toughness provides evidence that formalin preservation altered the bone, rendering it more brittle.

Figure 4 shows the time-change of bending strength. The bending strength of plexiform bone tended to diminish in most of the regions. Although almost no significant change is observed after 7 and 30 days of preservation, a significant decrease $(11 \%)$ is observed after 140 days of preservation. The bending strength of Haversian bone is significantly diminished in the formalin preservation group.

Burstein et $a .^{25)}$ and Wright et $a l .{ }^{26)}$ reported that yield stress, ultimate stress, and stiffness diminished in addition to Ca removal in cortical bone. Thus, the de-liming of inorganic components of bone is thought to affect mechanical parameters even in the case of formalin preservation.

Subsequently, the eluted elements in the preservative $(\mathrm{Ca}$, $\mathrm{P}$, and $\mathrm{Mg}$ ) from each group (7, 30, and 140 days of preservation) were quantitatively examined by ICP analysis.

Figure 5 shows the results of ICP analysis of eluted elements in plexiform bone and Haversian bone. The ordinate

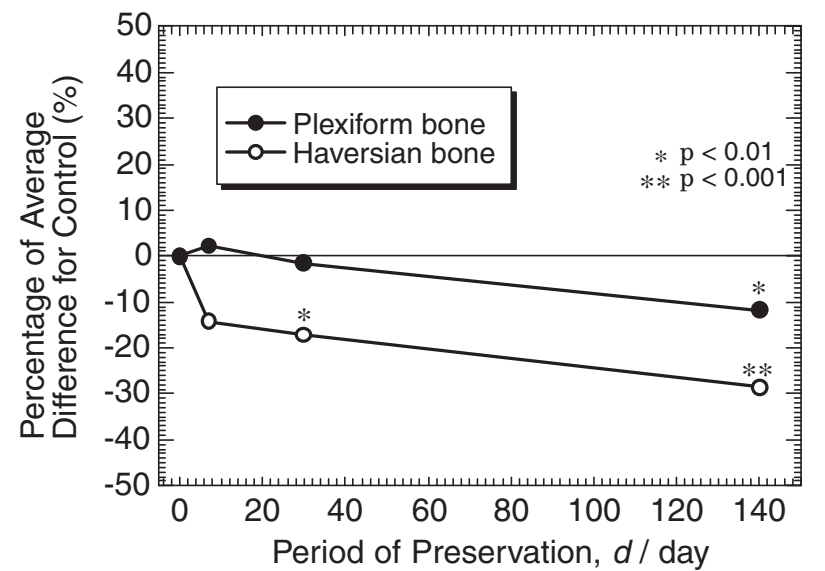

Fig. 4 Bending Strength.

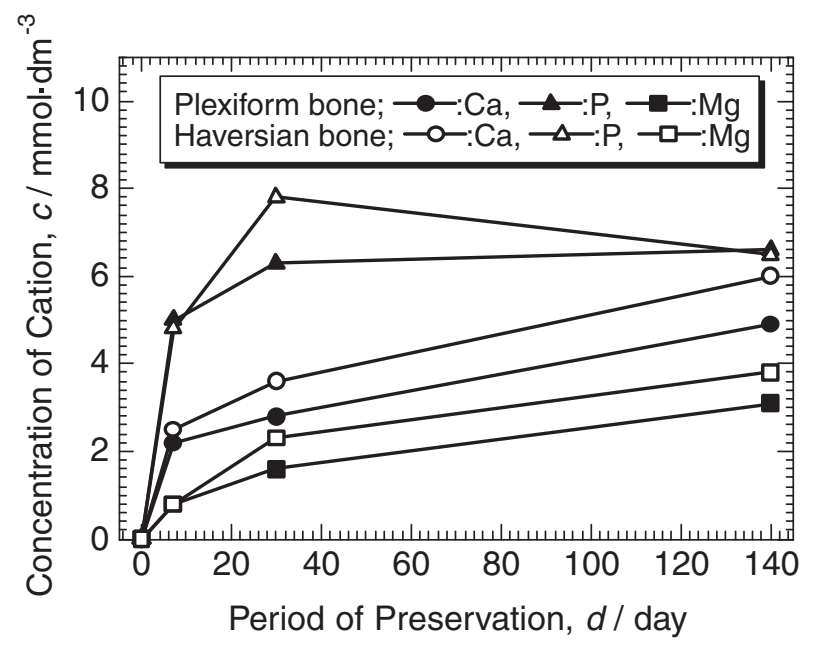

Fig. 5 Eluted mineral element concentrations in formalin.

indicates the eluted element concentration of formalin preservative, and abscissa indicates the preservation period.

Figure 5 indicates that eluted element concentration increases proportionally with the preservation period in plexiform bone and in Haversian bone, and the eluted element concentration was found to be higher in Haversian bone than in plexiform bone. This phenomenon can be explained as follows: $\mathrm{Ca}$ can be considered to elute as $\mathrm{Ca}^{2+}$, $\mathrm{P}$ as hydrogen phosphate ion $\left(\mathrm{H}_{2} \mathrm{PO}_{4}{ }^{-}, \mathrm{HPO}_{4}{ }^{2-}\right)$ or phosphate ion $\left(\mathrm{PO}_{4}{ }^{3-}\right)$, and $\mathrm{Mg}$ as $\mathrm{Mg}^{2+}$. Haversian bone and plexiform bone are composed of hydroxyapatite and collagen; however, the area fractions ${ }^{12)}$ of void of Haversian bone is higher than that of plexiform bone. Haversian bone offers a larger contact area of bone to the formalin preservative than does plexiform bone. This increases the probability of inorganic elements being eluting as ions in Haversian bone.

Comparison of results of bending strength shown in Fig. 4 and Fig. 5 shows that the elution quantity of $\mathrm{Ca}$ and $\mathrm{Mg}$ was augmented by formalin fixation while the bending strength was diminished. This implies that under formalin preservation, the strength of bone is dominated by the elution of $\mathrm{Ca}$ and $\mathrm{Mg}$.

On the other hand, the elution quantity of $\mathrm{P}$ differs from $\mathrm{Ca}$ and $\mathrm{Mg}$. Almost no change in eluted element concentration 
was observed in plexiform bone after 30 days of preservation, whereas in Haversian bone, the eluted element concentration reached a maximum on the 30th day of preservation, and later diminished gradually, finally achieving a value equivalent to plexiform bone on the 140th day of preservation. Such dissimilarity in change of elution quantities cannot be explained in the present situation.

The initial $\mathrm{pH}$ measurement of formalin preservative (0 days) was around 4 . The $\mathrm{pH}$ increased proportionally with the preservation period until day 7; subsequently, $\mathrm{pH}$ was saturated at around 7.9.

When bone is immersed in acidic formalin preservative, $\mathrm{Ca}^{2+}$ and $\mathrm{Mg}^{2+}$ are eluted and react with formalin preservative, and then neutralization is carried out. Phosphate and hydrogen phosphate ions in the preservative function as a buffer to stabilize the $\mathrm{pH}$ of the preservative at around 7 . Thus, the $\mathrm{pH}$ of preservative changes with prolonged preservation.

\section{Conclusion}

Antisepsis and sterilization of biomaterial using formalin are often performed, not only in medicine, but also in the measurement of the mechanical properties of bone. In spite of this, the effects of these chemical fixations on the material properties of bone have hardly been discussed in the literature.

This study specifically investigated the extent of change in the bending property and the fracture toughness of cortical bone induced by formalin preservation. In particular, we studied relatively long-term preservation, which has rarely been studied in the past.

Formalin is considered to harden collagen fibers in a relatively short period and to increase the brittleness of the entire bone. This is supported by the results of this experiment - formalin preservation augmented bending stiffness and diminished the fracture toughness of the bone. Inorganic components elute out from the bone in a relatively short period during formalin preservation, which is thought to contribute to the lowering of bending strength. Hydroxyapatite is reportedly difficult to alter by formalin. However, the results of this study revealed that formalin affects inorganic as well as organic components of a bone from the initial stage of preservation.

\section{REFERENCES}

1) J. McElhaney, J. Fogle, E. Byars and G. Weaver: J. Appl. Physiol. 19 (1964) 1234-1236.

2) F. G. Evans: Mechanical Properties of Bone, (Charles C. Thomas, Springfield, IL, 1973) pp. 56-60.

3) F. Linde and H. C. F. Sørensen: J. Biomech. 26 (1993) 1249-1252.

4) S. Tanaka, E. Watanabe, T. Hara, K. Hasegawa and H. Takahashi: Journal of Japanese Society for Clinical Biomechanics and Related Research 15 (1994) 1-6.

5) J. D. Currey, K. Brear, P. Zioupos and G. C. Reilly: Biomaterials 16 (1995) 1267-1271.

6) L. Strömberg and N. Dalén: Acta. Orthop. Scand. 47 (1976) 254-256.

7) R. R. Pelker, G. E. Friedlaender, T. C. Markham, M. M. Panjabi and C. J. Moen: J. Orthop. Res. 1 (1984) 405-411.

8) R. E. Borchers, L. J. Gibson, H. Burchardt and W. C. Hayes: Biomaterials 16 (1995) 545-551.

9) Q. Kang, Y. H. An and R. J. Friedman: Am. J. Vet. Res. 58 (1997) 1171-1174.

10) C. P. Boutros, D. R. Trout, M. Kasra and M. D. Grynpas: Vet. Comp. Orthop. Traumatol. 13 (2000) 59-64.

11) H. Kikugawa, H. Fukuda and Y. Yasui: Tokai J. Sports Med. Sci. 12 (2000) 62-66.

12) M. Niinoni, T. Akahori, J. Kim, H. Tajima and T. Kodama: Trans. Jpn. Soc. Mech. Eng. 69 (2003) 1641-1648.

13) S. P. Timoshenko and J. N. Goodier: Theory of Elasticity, (McGrawHill, 1970) pp. 113-122.

14) ASTM E399-90 (Reapproved 97).

15) T. L. Norman, D. Vashishth and D. B. Burr: J. Biomech. 25 (1992) 1489-1492.

16) K. Ichihara: Statistics for Bioscience, (Nankodo, 1994).

17) K. Yoshikawa, M. Mitsuishi, T. Nagao, H. Hashizume, H. Waki and K. Fujiwara: Journal of Japanese Society for Clinical Biomechanics and Related Research 20 (1999) 237-240.

18) K. Choi, J. L. Kuhn, M. J. Ciarelli and S. A. Goldstein: J Biomech. 23 (1990) 1103-1113.

19) D. T. Reilly and A. H. Burstein: The Journal of Bone and Joint Surgery 56A (1974) 1001-1022.

20) A. L. Boskey, M. L. Cohen and P. G. Bullough: Calcif. Tissue Int. 34 (1982) 328-331.

21) J. C. Behiri and W. Bonfield: J. Biomech. 17 (1984) 25-34.

22) J. C. Behiri and W. Bonfield: J. Biomech. 22 (1989) 863-872.

23) J. W. Melvin: J. Biomech. Eng. 115 (1993) 549-554.

24) T. L. Norman, D. Vashishth and D. B. Burr: J. Biomech. 28 (1995) 309-320.

25) A. H. Burstein, J. M. Zika, K. G. Heiple and L. Klein: The Journal of Bone and Joint Surgery 57A (1975) 956-961.

26) T. M. Wright, F. Vosburgh and A. H. Burstein: J. Biomech. 14 (1981) 405-409. 